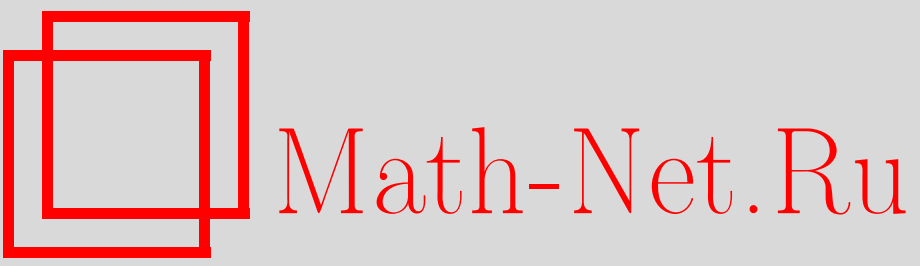

А. А. Алиева, Линейные отображения, монотонные относительно *-порядка Дрэйзина, УМН, 2003, том 58, выпуск 6, 145-146

DOI: https://doi.org/10.4213/rm679

Использование Общероссийского математического портала Math-Net.Ru подразумевает, что вы прочитали и согласны с пользовательским соглашением

http://www.mathnet.ru/rus/agreement

Параметры загрузки:

IP : 3.81 .55 .215

26 апреля 2023 г., 15:21:51 


\title{
ЛИНЕЙНЫЕ ОТОБРАЖЕНИЯ, МОНОТОННЫЕ ОТНОСИТЕЛЬНО $*$-ПОРЯДКА ДРЭЙЗИНА
}

\author{
А. А. АлиЕВА
}

Теория линейных отображений, сохраняющих различные матричные инварианты, свойства и отношения, восходит к результату Фробениуса, охарактеризовавшего линейные биективные отображения, сохраняющие определитель. В дальнейшем эта теория активно развивалась; подробный обзор основных результатов можно найти в [1]. K указанному классу задач относится исследование монотонных линейных преобразований матричной алгебры, т.е. линейных преобразований, сохраняющих то или иное отношение порядка на матричной алгебре. Настоящая работа посвящена исследованию линейных отображений на матричной алгебре, монотонных относительно *-порядка Дрэйзина.

Пусть $\mathbb{C}$ обозначает поле комплексных чисел, $M_{m, n}(\mathbb{C})$ - пространство матриц размера $m \times n$ над $\mathbb{C}, E_{i, j}$ - матричную единицу, $A^{*}$ - транспонированную комплексно-сопряженную матрицу к матрице $A$. Определим *-порядок Дрэйзина на $M_{m, n}(\mathbb{C})$ следующим образом, см. [2].

ОПредЕЛЕние 1. Пусть матрицы $A, B \in M_{m, n}(\mathbb{C})$. Тогда говорят, что $A \leqslant B$, если $A^{*} A=A^{*} B$ и $A A^{*}=B A^{*}$.

Теорема 1. Пусть линейное отображение $T: M_{m, n}(\mathbb{C}) \rightarrow M_{m, n}(\mathbb{C})$ монотонно относительно *-порядка Дрәйзина, тогда либо $T \equiv 0$, либо Т биективно.

ДокАЗАТЕльство. Рассмотрим полугруппу $G$, порожденную отображением $T$ и всеми отображениями вида $X \rightarrow \alpha M X N$ или, при $m=n, X \rightarrow \alpha M X^{t} N$, где $\alpha \in \mathbb{C}, M, N$ - унитарные матрицы. Выберем в $G$ такой элемент $L$, что $\operatorname{rk} L\left(E_{1,1}\right)=s$, для любого отображения $L^{\prime} \in G$ справедливо $\operatorname{rk} L^{\prime}\left(E_{1,1}\right) \leqslant s$ и для любой матрицы $A \in M_{m, n}(\mathbb{C}), \operatorname{rk} A=1$, справедливо $\operatorname{rk} L(A) \leqslant s$. Такое $L$ существует, поскольку, пользуясь сингулярным разложением, для любой матрицы $A \in M_{m}, n(\mathbb{C}), \operatorname{rk} A=1$, можно найти такие $\alpha \in \mathbb{C}$ и унитарные матрицы $M, N$, что $\alpha M A N=E_{1,1}$ и отображение $X \rightarrow \alpha M X N$ монотонно относительно $*$-порядка Дрэйзина в силу [3].

Будем доказывать, что $s=1$. Предположим, что $s \geqslant 2$. Покажем, что в этом случае $L\left(E_{1,1}\right)=0$, что приведет нас к противоречию. По теореме о сингулярном разложении существуют такие $\alpha \in \mathbb{C}$ и унитарные матрицы $U \in M_{m, m}(\mathbb{C})$ и $V \in M_{n, n}(\mathbb{C})$, что $\alpha U L\left(E_{1,1}\right) V=E_{1,1}+\alpha_{2} E_{2,2}+\cdots+\alpha_{s} E_{s, s}, \alpha_{2} \cdots \alpha_{s} \neq 0$. Так как

$$
E_{1,1}<^{*} E_{1,1}+\alpha_{2} E_{2,2}<^{*} \cdots<^{*} E_{1,1}+\alpha_{2} E_{2,2}+\cdots+\alpha_{s} E_{s, s},
$$

то

$$
L\left(E_{1,1}\right)<{ }^{*} L\left(E_{1,1}+\alpha_{2} E_{2,2}\right)<* \cdots<{ }^{*} L\left(E_{1,1}+\alpha_{2} E_{2,2}+\cdots+\alpha_{s} E_{s, s}\right),
$$

и, следовательно, в силу [4], имеем:

$$
s=\operatorname{rk} L\left(E_{1,1}\right) \leqslant \operatorname{rk} L\left(E_{1,1}+\alpha_{2} E_{2,2}\right) \leqslant \cdots \leqslant \operatorname{rk} L\left(E_{1,1}+\alpha_{2} E_{2,2}+\cdots+\alpha_{s} E_{s, s}\right) .
$$

Пусть $\mathrm{rk} L\left(E_{1,1}+\alpha_{2} E_{2,2}\right)>s$, тогда и $\operatorname{rk} L\left(E_{1,1}+\alpha_{2} E_{2,2}+\cdots+\alpha_{s} E_{s, s}\right)>s$, т.е. получаем $\operatorname{rk} L\left(\alpha U L\left(E_{1,1}\right) V\right)>s$, что противоречит выбору $L$. Следователшно, $\operatorname{rk} L\left(E_{1,1}+\alpha_{2} E_{2,2}\right)=s$ и поэтому $L\left(E_{2,2}\right)=0$, так как в силу [4; следствие 1.6$]$ из одновременного вьполнения условий $A<{ }^{*} B$ и rk $A=\operatorname{rk} B$ следует, что $A=B$.

Далее, пусть $A$ - такая $(s \times s)$-подматрица в $L\left(E_{1,1}\right)$, что $\operatorname{det} A \neq 0$ (она существует, так как $\left.\operatorname{rk} L\left(E_{1,1}\right)=s\right)$. Пусть $B$ - подматрица матрицы $L\left(E_{1,2}+E_{2,1}\right)$, расположенная на пересечении тех же строк и столбцов, что и $A$. Существуют невырожденные матрицы $S, T \in M_{s, s}(\mathbb{C})$ такие, что $\operatorname{det}(S T)=1$ и $S A T=E_{1,1}+\cdots+E_{s-1, s-1}+(\operatorname{det} A) E_{s, s}$. Тогда

$$
\operatorname{det}(x A+B)=\operatorname{det}(\operatorname{diag}(x, \ldots, x, x \operatorname{det} A)+S B T)=x^{s} \operatorname{det} A+\cdots
$$

- полином степени $s$, поэтому существуют ненулевые $x_{1} \neq x_{2} \in \mathbb{C}$ такие, что $\operatorname{det}\left(x_{i} A+B\right) \neq 0$ и, следовательно, $\operatorname{rk} L\left(x_{i} E_{1,1}+E_{1,2}+E_{2,1}\right) \geqslant s, i=1,2$. Для любого $x \neq 0$ имеем:

$$
\left(\begin{array}{ccc}
x & 1 & \overrightarrow{0}^{t} \\
1 & \frac{1}{x} & \overrightarrow{0}^{t} \\
\overrightarrow{0} & \overrightarrow{0} & 0
\end{array}\right)<^{*}\left(\begin{array}{ccc}
x+\frac{1}{x} & 0 & \overrightarrow{0}^{t} \\
0 & x+\frac{1}{x} & \overrightarrow{0}^{t} \\
\overrightarrow{0} & \overrightarrow{0} & 0
\end{array}\right) .
$$


Таким образом, так как $L$ монотонно и $L\left(E_{2,2}\right)=0$, имеем: $L\left(x E_{1,1}+E_{1,2}+E_{2,1}\right)=L\left(x E_{1,1}+\right.$ $\left.E_{1,2}+E_{2,1}+\frac{1}{x} E_{2,2}\right) \leqslant{ }^{*} L\left(\left(x+\frac{1}{x}\right) E_{1,1}+\left(x+\frac{1}{x}\right) E_{2,2}\right)=L\left(\left(x+\frac{1}{x}\right) E_{1,1}\right)$. В силу выбора $x_{1}, x_{2}$ для $x=x_{i}, i=1,2$, имеем: $\operatorname{rk} L\left(x E_{1,1}+E_{1,2}+E_{2,1}\right) \leqslant \operatorname{rk} L\left(\left(x+\frac{1}{x}\right) E_{1,1}\right)=s$. Следовательно, в силу [ 4 ; следствие 1.6], справедливо $L\left(x_{i} E_{1,1}+E_{1,2}+E_{2,1}\right)=L\left(\left(x_{i}+\frac{1}{x_{i}}\right) E_{1,1}\right)$, т.е. $L\left(E_{1,2}+\right.$ $\left.E_{2,1}\right)=\frac{1}{x_{i}} L\left(E_{1,1}\right), i=1,2$. Отсюда, так как $x_{1} \neq x_{2}$, имеем $L\left(E_{1,1}\right)=0$-противоречие с $s \geqslant 2$.

Таким образом, $s \leqslant 1$. Если $s=0$, то, очевидно, $T \equiv 0 ;$ пусть теперь $s=1$. Тогда в силу [5] имеем следующие два случая.

1. $\operatorname{Im}(L) \subseteq\left\{A \in M_{m, n}(\mathbb{C}) \mid \operatorname{rk} A \leqslant 1\right\}$. Так как $E_{1,1}<^{*} E_{1,1}+E_{2,2}$, то $L\left(E_{1,1}\right)<*$ $L\left(E_{1,1}+E_{2,2}\right)$, и, следовательно, $1=\operatorname{rk} L\left(E_{1,1}\right) \leqslant \operatorname{rk} L\left(E_{1,1}+E_{2,2}\right) \leqslant 1$. Получаем $L\left(E_{2,2}\right)=$ 0 и далее аналогично случаю $s \geqslant 2$ получаем $L\left(E_{1,1}\right)=0$, что противоречит условию $s=1$.

2. Существуют матрицы $P \in M_{m, m}(\mathbb{C})$ и $Q \in M_{n, n}(\mathbb{C})$ такие, что $L(X)=P X Q$ для всех $X \in M_{m, n}(\mathbb{C})$ или $m=n$ и $L(X)=P X^{t} Q$ для всех $X \in M_{m, n}(\mathbb{C})$.

Пусть $L(X)=P X Q$. Существуют $\beta, \gamma \in \mathbb{C}$ и унитарные матрицы $R_{0}, R_{1} \in M_{m}, m(\mathbb{C})$ и $S_{0}, S_{1} \in M_{n, n}(\mathbb{C})$ такие, что $P=\beta R_{0} U S_{0}, Q=\gamma R_{1} V S_{1}$, где $U=\operatorname{diag}\left(1, u_{2}, \ldots, u_{m}\right)$, $V=\operatorname{diag}\left(1, v_{2}, \ldots, v_{n}\right)$. Таким образом, $L$ является композицией трех отображений: $L_{1}(X)=$ $\gamma S_{0} X R_{1}, L_{2}(X)=U X V, L_{3}(X)=\beta R_{0} X S_{1}$. Из монотонности $L$ и вида отображений $L_{1}$ и $L_{3}$ следует монотонность отображения $L_{2}$. Имеем $L_{2}\left(E_{i, j}\right)=u_{i} v_{j} E_{i, j}$. Легко проверить, что $E_{i, j}+E_{i, j+1}+E_{i+1, j}+E_{i+1, j+1}<^{*} 2 E_{i, j}+2 E_{i+1, j+1}$, и таким же неравенством связаны образы этих матриц при отображении $L_{2}$, т.е. $C=u_{i} v_{j} E_{i, j}+u_{i} v_{j+1} E_{i, j+1}+u_{i+1} v_{j} E_{i+1, j}+$ $u_{i+1} v_{j+1} E_{i+1, j+1}<^{*} 2 u_{i} v_{j} E_{i, j}+2 u_{i+1} v_{j+1} E_{i+1, j+1}=D$. Тогда по определению из $C C^{*}=D C^{*}$ имеем: $\left|u_{i}\right|^{2}\left(\left|v_{j}\right|^{2}+\left|v_{j+1}\right|^{2}\right) E_{i, i}+u_{i} \bar{u}_{i+1}\left(\left|v_{j}\right|^{2}+\left|v_{j+1}\right|^{2}\right) E_{i, i+1}+\bar{u}_{i} u_{i+1}\left(\left|v_{j}\right|^{2}+\right.$ $\left.\left|v_{j+1}\right|^{2}\right) E_{i+1, i}+\left|u_{i+1}\right|^{2}\left(\left|v_{j}\right|^{2}+\left|v_{j+1}\right|^{2}\right) E_{i+1, i+1}=2\left|u_{i}\right|^{2}\left|v_{j}\right|^{2} E_{i, i}+2 u_{i} \bar{u}_{i+1}\left|v_{j}\right|^{2} E_{i, i+1}+$ $2 \bar{u}_{i} u_{i+1}\left|v_{j+1}\right|^{2} E_{i+1, i}+2\left|u_{i+1}\right|^{2}\left|v_{j+1}\right|^{2} E_{i+1, i+1}$, откуда $\left|u_{i}\right|^{2}\left|v_{j}\right|^{2}=\left|u_{i}\right|^{2}\left|v_{j+1}\right|^{2}$. Так как $u_{1}=v_{1}=1$, то получаем $\left|v_{2}\right|=1$. Аналогично, рассматривая равенство $C^{*} C=C^{*} D$, получаем $\left|u_{i}\right|^{2}\left|v_{j}\right|^{2}=\left|u_{i+1}\right|^{2}\left|v_{j}\right|^{2}$, откуда $\left|u_{2}\right|=1$. Действуя таким образом, получаем $\left|u_{i}\right|=1,1 \leqslant i \leqslant m,\left|v_{j}\right|=1,1 \leqslant j \leqslant n$. Значит, матрицы $U$ и $V$ унитарны, т.е. $L$, а следовательно, и $T$ биективно.

Случай $L(X)=P X^{t} Q$ рассматривается аналогично.

Применяя доказанную теорему вместе с классификационной теоремой из [3], получаем следующий резултат:

Tеорема 2. Пусть $T: M_{m, n}(\mathbb{C}) \rightarrow M_{m, n}(\mathbb{C})$ - линейное отображение. Oтображение $T$ монотонно относительно *-порядка Дрэйзина тогда и только тогда, когда существуют такие унитарные матрицы $P, Q \in M_{m, n}(\mathbb{C})$ и (возможно, нулевая) константа $\alpha \in \mathbb{C}$, что $T(X)=\alpha P X Q$ для всех $X \in M_{m, n}(\mathbb{C})$ или $T(X)=\alpha P X^{t} Q$ для всех $X \in M_{m, n}(\mathbb{C})$ npu $m=n$.

\section{СПИСОК ЛИТЕРАТУРЫ}

[1] C.-K. Li, S. Pierce, N.-K. Tsing (Eds.). A Survey of Linear Preserver Problems. Taylor \& Francis, 1992. (Linear and Multilinear Algebra. V. 33. № 1-2 (Special issues).) [2] M.P. Drazin // Bull. Amer. Math. Soc. 1978. V. 84. №1. P. 139-141. [3] A. Guterman // Comm. Algebra. 2001. V. 29. № 9. P. 3905-3917. [4] R. E. Hartwig, R. Loewy // Linear Algebra Appl. 1992. V. 175. P. 39-61. [5] P. Botta // Linear and Multilinear Algebra. 1987. V. 20. № 3. P. 197-201. 